\title{
Intracranial hypotension syndrome: a comprehensive review
}

\author{
Michael Paldino, M.D., Alon Y. Mogilner, M.D., Ph.D., and Michael S. Tenner, M.D. \\ Departments of Neurosurgery and Radiology, New York Medical College, Valhalla and New York, \\ New York
}

\begin{abstract}
Intracranial hypotension may have variable clinical presentations, but has a rather uniform component of postural headache among its symptomatology. Its symptoms are explainable given the effects of the hypotension and attempts within the craniospinal axis to maintain volume homeostasis in the face of cerebrospinal fluid leakage (Monro-Kellie hypothesis). The imaging corollaries of the consequences of intracranial hypotension are especially well depicted on magnetic resonance imaging studies.
\end{abstract}

\section{KEY WORDS - intracranial hypotension - postural headache • spontaneous subdural collection $\bullet$ pituitary gland}

The syndrome of ICH is a single pathophysiological entity of diverse origin. Usually, it is characterized by an orthostatic headache, that is, one that occurs or worsens with upright posture, although patients with chronic headaches or even no headache have been described. ${ }^{31}$ The nature and location of the headache vary greatly from patient to patient; but consistently the pain is exacerbated by laughing, coughing, jugular venous compression, and Valsalva maneuver, and is resistant to treatment with analgesic agents. ${ }^{24,42}$ In addition to headache, patients may experience nausea, vomiting, anorexia, neck pain, dizziness, horizontal diplopia, changes in hearing, galactorrhea, facial numbness or weakness, or radicular symptoms involving the upper limb, all of which are orthostatic in nature ${ }^{36}$ Intracranial hypotension generally is considered to be a benign condition, and most cases resolve with conservative management. With advances in diagnosis, however, atypically disabling presentations are increasingly recognized including parkinsonism, frontotemporal dementia, syringomyelia, hypopituitarism, seizures, coma, and death. ${ }^{4,11,16,18,24,49}$

The diagnosis of ICH usually can be confirmed by demonstrating decreased CSF opening pressure, often less than $60 \mathrm{~mm} \mathrm{H}_{2} \mathrm{O}$, on performing an LP. In some cases, spinal pressure may be so low that Valsalva maneuver or gentle aspiration with a syringe is required to produce CSF. ${ }^{4}$ Rarely, a "sucking noise" has been described to oc-

Abbreviations used in this paper: $\mathrm{ICH}=$ intracranial hypotension; $\mathrm{CSF}=$ cerebrospinal fluid; $\mathrm{CT}=$ computerized tomography; $\mathrm{LP}=$ lumbar puncture; $\mathrm{MR}=$ magnetic resonance; $\mathrm{RC}=$ radioisotope cisternography. cur as the stylet is withdrawn and air enters the subarachnoid space, indicating subatmospheric pressure. ${ }^{47}$ Analysis of the CSF may show it to be entirely within normal limits, but more often increased protein concentration, lymphocytic pleocytosis, increased erythrocyte count, and/or xanthochromia are identified at some point during the patient's illness. ${ }^{11}$ Cultures of CSF are always nondiagnostic, and glucose levels are never low. ${ }^{35}$

There is strong evidence indicating that most cases of ICH result from a persistent CSF leak. Such a leak most commonly occurs after dural puncture for a diagnostic LP, myelography, or spinal anesthesia. Nonetheless, symptoms of ICH may be experienced any time the dura mater is violated, such as after craniotomy, spinal surgery, craniospinal trauma, or placement of a ventriculoperitoneal shunt. ${ }^{26}$ In some cases, this syndrome may occur in the absence of an identifiable precipitant and, in fact, is believed to have developed spontaneously. Medical causes of ICH include dehydration, diabetic coma, hyperpnea, uremia, and severe systemic illness. ${ }^{7}$ Loss of CSF caused by the formation of a thoracomeningeal fistula is a rare complication of thoracic surgery. ${ }^{2}$

Advances in neuroimaging have improved our ability to diagnose ICH. In particular, findings on MR imaging studies that are characteristic of this syndrome have allowed physicians to appreciate its true incidence as well as its varied modes of presentation. Here we review the role that imaging modalities play in the diagnosis of ICH and the pathophysiological mechanisms that underlie its symptomatology, clinical findings, and neuroimaging abnormalities. Specific causes as well as treatment options will also be considered. 


\section{IMAGING STUDIES}

\section{Cranial MR Imaging}

Magnetic resonance imaging has revolutionized the diagnosis of ICH. The major abnormalities demonstrated on MR imaging studies in patients with ICH are diffuse thickening of the pachymeninges with Gd enhancement, engorgement of venous sinuses, subdural fluid collections, enlargement of the pituitary gland, and downward displacement of the brain. ${ }^{32}$ The resolution of these abnormalities on MR images parallels improvement in clinical symptoms. ${ }^{46}$

Evidence indicates that several of these abnormalities are the result of vascular dilation to compensate for reduced CSF volume. ${ }^{12}$ This idea is based on the application of the Monro-Kellie hypothesis, which states that the sum of the volumes of intracranial blood, CSF, and brain tissue must remain constant in an intact cranium. According to this hypothesis, increased intracranial blood volume compensates for acute loss of CSF. Most of this compensation occurs through dilation of the venous side of the circulation, given its greater compliance and capacitance. Thus, venous sinus engorgement, abnormal pachymeningeal enhancement, subdural effusions, and enlargement of the pituitary gland occurring in ICH may represent compensatory changes to maintain intracranial volume in the face of CSF loss.

Meningeal enhancement in ICH is thick, linear, without nodularity, and involves the pachymeninges of both the infra- and supratentorial compartments without evidence of involvement of the leptomeninges (no abnormal enhancement around the brainstem, within the sylvian fissures, or in the depth of cerebral sulci). ${ }^{9}$ The innermost layer of the dura mater has no collagen fibers and, instead, is composed of dural border cells, that is, fibroblasts with interdigitating processes that create spaces of various shapes and sizes..$^{34}$ The dura mater does not contribute to the blood-brain barrier and therefore lacks tight junctions. Consequently, the pressure gradient established by increased volume in the dural vasculature causes extravasation of fluid into this innermost dural border-cell layer. ${ }^{35}$ Venous engorgement thus results in a greater concentration of contrast not only in the dural vasculature, but also in the interstitium. The venous engorgement occurring with this entity has been emphasized in recent articles. ${ }^{20,45}$ Enlargement of dural arteries and medullary and cortical veins were reported to be the expected consequences of the intracranial-intraspinal volume maintenance required by the Monro-Kellie hypothesis. Tight junctions in microvessels of the arachnoid and pia mater prevent similar contrast accumulation in these layers, explaining why $\mathrm{Gd}$ enhancement on MR imaging is limited to the dura.

Subdural effusions are typically thin, crescentic, and located either below or between enhancing membranes. ${ }^{39}$ They may be bilateral and are usually without mass effect. ${ }^{34}$ Effusions occur when the aforementioned extravasation continues to the point of fluid accumulation in the subdural space. Such collections correlate with the most significant meningeal thickening. ${ }^{6}$ Furthermore, in a study by Brightbill, et al., ${ }^{6}$ effusions were not seen in the absence of meningeal enhancement. Taken together, these results raise the possibility that subdural fluid collections may occur only in cases in which dural venous dilation alone is insufficient to maintain intracranial volume. Thus, subdural fluid collection may represent a greater degree of fluid accumulation in the dural border-cell layer to compensate for more severe CSF volume loss.

The pituitary gland is a densely vascularized organ surrounded by the cavernous sinus. ${ }^{17}$ Its size appears larger on MR images during the symptomatic phase of ICH compared with after recovery. ${ }^{3}$ In fact, on MR imaging studies, evidence of gland enlargement has been shown to reverse earlier than meningeal thickening. Like venous engorgement, increased meningeal enhancement, and subdural fluid collections, this increase in volume presumably reflects compensatory venous hyperemia. ${ }^{17}$

Gross and microscopic features of meningeal biopsies obtained from patients with ICH and Gd enhancement on MR imaging are consistent with these conclusions. Sections have demonstrated a normal appearance of the periosteal and meningeal (outermost) layers of dura. ${ }^{34}$ Nonetheless, they have revealed a membrane of fibroblasts and small, thin-walled blood vessels in an amorphous matrix corresponding to the layer of the dural border cells, the same layer where subdural effusions occur. ${ }^{35}$ That abnormal findings are limited to this structurally weak layer is consistent with the idea that contrast enhancement on MR imaging results from pressure-driven extravasation of fluid from engorged dural veins. The absence of inflammatory cells further supports the theory that these meningeal abnormalities represent reactive changes caused by venous engorgement. ${ }^{35}$

Some groups have demonstrated meningeal fibrosis on biopsy. ${ }^{14}$ Based on these findings, meningeal enhancement on MR imaging has been attributed to inflammation of the pachymeninges. Indeed, an increase in leptomeningeal connective tissue elements and proliferation of arachnoid cap cells have been noted in some patients with $\mathrm{ICH} .{ }^{33}$ Fishman and Dillon ${ }^{12}$ have attributed the meningeal fibrosis seen on such biopsies to nonspecific reactive change caused by chronic venous congestion, however. This idea is consistent with the work of Mokri and colleagues ${ }^{34,35}$ who found such abnormalities only in patients with the most prolonged symptoms. Data collected by these investigators have consistently demonstrated a lack of inflammatory cells in all meningeal specimens. ${ }^{34}$ Thus, pachymeningeal enhancement and late meningeal fibrosis may both be the result of venous engorgement caused by $\mathrm{ICH}$.

Although it is a frequent finding, abnormal meningeal enhancement in patients with ICH is not the absolute rule. Mokri, et al., ${ }^{32}$ documented enhancement that resolved while the patients were still symptomatic, a patient without enhancement on initial studies, and patients whose MR images never revealed enhancement. Thus, it seems that Gd enhancement varies during the course of a patient's illness. Furthermore, in some patients, the loss of volume and hydrostatic pressure changes may never be sufficient to result in venous congestion significant enough to lead to the appearance of meningeal enhancement on MR images. ${ }^{7}$

Imaging evidence of descent of the brain includes the following: descent of cerebellar tonsils, which may be mistaken for an Arnold-Chiari malformation Type I; 
reduction in size or effacement of the prepontine cistern; inferior displacement of the optic chiasm; effacement or obliteration of perichiasmatic cisterns; reduction of subarachnoid cisterns; and descent of the iter (opening of the sylvian aqueduct on MR imaging) judged relative to the incisural line (connecting the anterior tuberculum sellae to the point of confluence of straight and inferior sinuses and the great cerebral vein). ${ }^{35}$ The buoyant action of CSF reduces the effective weight of the brain to less than 50 g. ${ }^{33}$ Loss of this buoyant force allows the brain to sag, which is seen as evidence of descent of the brain on MR imaging. Alternately, downward displacement could be caused by downward force exerted by subdural effusions. Most collections are small, however, without mass effect or evidence of compression of underlying sulci, and not under pressure at evacuation, thus casting doubt on such a causal role. ${ }^{32,39}$ One group of researchers has found downward displacement of the brain only in patients with the most severe CSF volume depletion, indicating that it may occur after the compensatory mechanisms described earlier have been exhausted. ${ }^{6}$ The supine position of the patient during MR imaging may lead investigators to underestimate imaging evidence of descent.

Another complication of the syndrome of ICH that appears as a subdural fluid collection on MR imaging is a subdural hematoma. The cause of such hematomas is presumably a rupture of the bridging veins as the CSF volume decreases and the brain sags, pulling away from the dura. ${ }^{24}$ This idea is consistent with work of Grant, et al., ${ }^{15}$ who found that the majority of reduced CSF volume can be explained by loss of fluid over the cerebral sulci.

Case Illustration. This 37-year-old woman presented with symptoms of postural headache, double vision, and lowback pain. Results of MR imaging studies obtained at the time that she was symptomatic revealed enlargement of the pituitary gland, diffuse dural enhancement, mild narrowing of the ventricular system and cerebral sulci, and mild descent of the brainstem and cerebellum (Fig. 1). She responded to conservative therapy without the need for a blood patch and remains asymptomatic more than 3 months later (Fig. 2).

\section{Radioisotope Cisternography}

Cerebrospinal fluid is produced mainly by the choroid plexus. The pulse pressure from the choroid plexus drives CSF out of the ventricular system via the foramina of Magendie and Luschka into the vertebral dural sac. The CSF then proceeds upward around the hemispheres and is absorbed by the arachnoid villi into the sagittal sinus.

Radioisotope cisternography is a useful procedure for identifying CSF leaks. During such an investigation, a radioactive tracer is injected into the lumbar subarachnoid space. Scanning of the entire neuraxis by using a gamma camera allows observation of the pattern of radioactivity over time. ${ }^{24}$ Under conditions of normal CSF flow, the tracer migrates cephalad from the site of injection to the cerebral convexities and sylvian fissure, where it is absorbed into the bloodstream. ${ }^{26} \mathrm{~A}$ CSF leak can be detected either directly by accumulation of radioactivity outside the subarachnoid space or indirectly by a limited ascent of the tracer to the cerebral convexities and its early accumulation in the kidneys, urinary bladder, and soft tissues. ${ }^{35}$ These findings are most consistent with CSF leakage from the subarachnoid space and absorption via the epidural paravertebral venous plexus. Cerebrospinal fluid leaks not detected directly are presumed to represent an isotope loss smaller than the minimum resolution of this test, perhaps escaping directly into the epidural venous plexus and thus being removed too rapidly to be perceived. ${ }^{23}$ Given that this test yields positive findings only if an active CSF leak is present, intermittent leaks may remain undetected. Note that leaks are most commonly demonstrated in the thoracic region of the vertebral column. ${ }^{46}$

Initially, findings on RC were interpreted as evidence that ICH results from CSF hyperabsorption at the arachnoid villi; ${ }^{21,26}$ however, the characteristic finding of rapid isotope loss despite a lack of ascent to the convexities, the location of CSF absorption, makes this possibility less likely. Furthermore, research data have repeatedly demonstrated that reabsorption of CSF at the arachnoid villi does not occur below a critical pressure of approximately 60

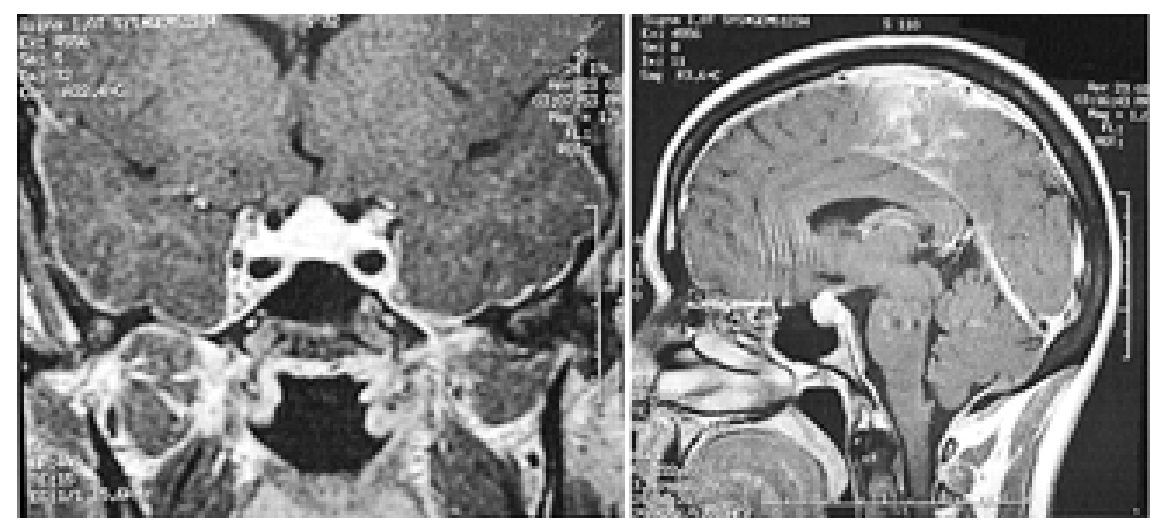

Fig. 1. Magnetic resonance images obtained in a patient with $\mathrm{ICH}$ before treatment. Left: Coronal post-Gd $\mathrm{T}_{1}-$ weighted image of a 3-mm-thick section of the brain revealing enlargement of the pituitary gland and dural enhancement of the visualized middle fossa. Center: Sagittal post-Gd $\mathrm{T}_{1}$-weighted 3-mm-thick section demonstrating enlargement of the pituitary gland, dural enhancement along the clivus, and mild descent of the brainstem and cerebellum. Right: Axial post-Gd $\mathrm{T}_{1}$-weighted image of a 5-mm-thick section exhibiting diffuse dural enhancement and mild narrowing of the lateral ventricles and cerebral sulci. 

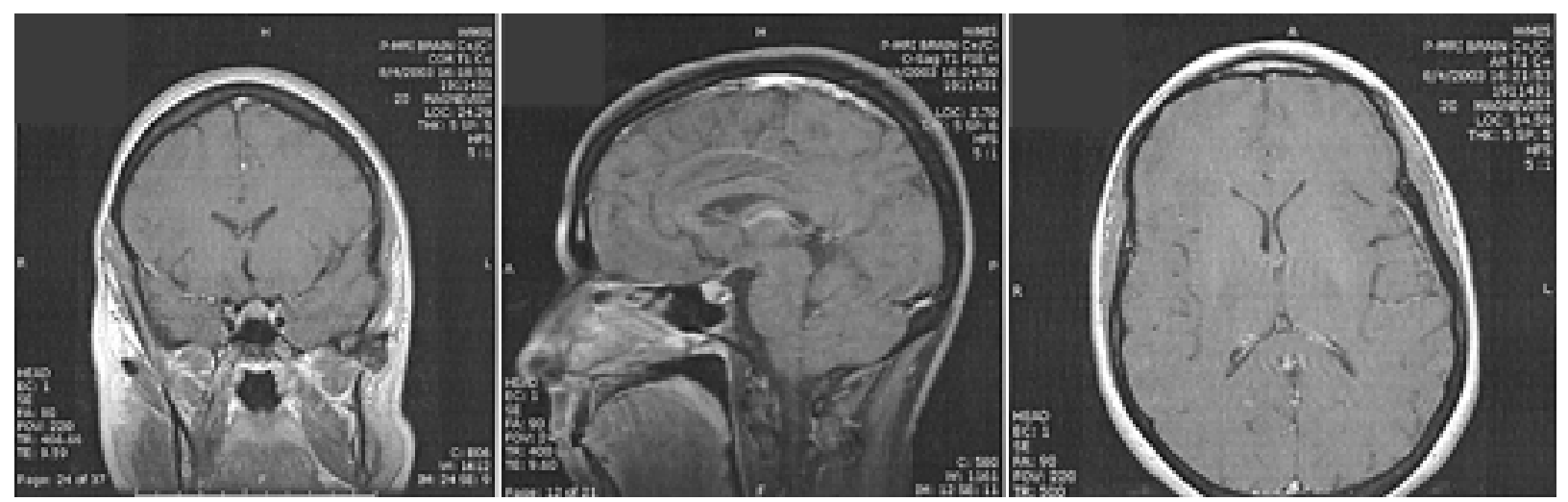

Fig. 2. Magnetic resonance images obtained in the same patient featured in Fig. 1 at a follow-up visit 3.5 months later. Left: Coronal post-Gd $\mathrm{T}_{1}$-weighted image of a 3-mm-thick section of the brain revealing that the pituitary gland has decreased in size and the dural enhancement has resolved. Center: Sagittal post-Gd T $\mathrm{T}_{1}$-weighted image of a 3-mm-thick section revealing that the pituitary gland has decreased in size, the dural enhancement of the clivus has resolved, and the brainstem descent has resolved. Right: Axial post-Gd $\mathrm{T}_{1}$-weighted image of a 5-mm-thick section indicating that the dural enhancement has resolved and the lateral ventricles and cerebral sulci have expanded.

mm $\mathrm{H}_{2} \mathrm{O}$, a level higher than that in most patients with $\mathrm{ICH} .{ }^{23}$ Nonetheless, a CSF opening pressure lower than the normal range is not an absolute in the syndrome of $\mathrm{ICH} .{ }^{31}$

The sensitivity of RC is not well documented. Benamor, et al., ${ }^{5}$ successfully used this technique to detect a CSF leak directly only $60 \%$ of the time. In another study, however, RC demonstrated at least one abnormal finding in $91 \%$ of cases of ICH. ${ }^{9}$ Thus, even when RC does not show the level of the leak, the technique almost always provides indirect evidence for its presence and supports the diagnosis of ICH..${ }^{35}$ Recently, Inenaga, et al., ${ }^{19}$ reported the use of a combination of an upright patient position and breath holding to increase the sensitivity of RC to detect CSF leaks directly. This technique is a sort of Valsalva maneuver, increasing intracranial and spinal CSF pressure, and thus might accelerate a CSF leak to a minimally detectable level. ${ }^{28}$ Alternatively, a check-valve mechanism, in which leakage occurs only above a certain critical pressure, may explain why certain leaks can be detected only with this technique. ${ }^{19}$

\section{Computerized Tomography of the Brain}

Computerized tomography of the brain often demonstrates obliteration of the basilar cisterns due to sagging of the brain. This could be misdiagnosed as subarachnoid hemorrhage. The dural thickening changes are infrequently found, although subdural hemorrhages are easily seen.

\section{Computerized Tomography Myelography}

Computerized tomography myelography has been used to demonstrate CSF leaks. In this procedure, CT slices are obtained through the skull base and along the entire spinal axis at each spinal level after administering an intrathecal injection of an iodinated contrast agent. The greater resolution of CT offers several advantages. First, leaks can be localized more precisely. In addition, underlying anatomical defects responsible for the leak, including meningeal diverticula, can be revealed. ${ }^{46}$
Research data indicate that CT myelography is the study of choice for localization of such leaks. In a study by Mokri, et al. ${ }^{35} \mathrm{CT}$ myelography demonstrated the level of a CSF leak in $67 \%$ of patients overall compared with only 50 and $55 \%$ for spinal MR imaging and $\mathrm{RC}$, respectively. Of the six patients who had undergone both CT myelography and spinal MR imaging, CT myelography demonstrated the source of the leak in five patients whereas spinal MR imaging revealed the source only in three. Both CT myelography and RC were performed in nine patients-leaks were demonstrated in four by $\mathrm{RC}$ and in six by CT myelography. In no case did RC reveal the level of the leak when CT myelography did not. When both tests demonstrated the leak, the level of involvement was more precisely identified on CT myelography.

These results are consistent with the findings of Schievink, et al. ${ }^{46}$ Of 10 patients, RC failed to demonstrate CSF leaks revealed on CT myelography in three, a false-negative rate of $30 \%$. Similarly, spinal MR imaging results were nondiagnostic in two of six patients with previously documented CSF leaks.

Despite these findings, the sensitivity of CT myelography has not been well studied, and many CSF leaks remain occult on all types of spinal imaging studies. Furthermore, CT myelography performed without the benefit of a previous imaging study to demonstrate the approximate location of a leak requires that CT slices be obtained through the skull base and throughout the entire spinal axis, a difficult and time-consuming process. ${ }^{27}$ Therefore, spinal MR imaging and RC may serve as guides for obtaining cuts on CT myelography only at the appropriate levels.

\section{Spinal MR Imaging}

Recently, MR imaging has begun to be used to elucidate spinal imaging findings associated with ICH. The cardinal MR imaging findings include extraarachnoid fluid collections, spinal meningeal enhancement, and dilation of the anterior internal vertebral (epidural) venous plexus. ${ }^{30}$ 
With the use of this technique, many patients with contrast-enhancing extraarachnoid fluid collections in the spine have been identified. ${ }^{44}$ Computerized tomography myelography and RC have been used to document direct communication between such collections and the thecal sac. ${ }^{40}$ Taken together, these results have been interpreted by some investigators as evidence that spinal fluid collections detected on MR imaging are due to CSF leakage and

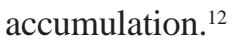

Increased protein concentration and cell count of this fluid combined with contrast enhancement of these collections have led some researchers to assert that these findings may represent transudation of fluid out of the subarachnoid space as a result of venous hyperemia. ${ }^{6}$ Consistent with this hypothesis, spinal MR imaging has demonstrated meningeal enhancement as well as dilation of the anterior internal vertebral (epidural) venous plexus. ${ }^{644}$ Thus, the same principles likely governing intracranial venous engorgement and extraarachnoid fluid collection, described previously as the Monro-Kellie hypothesis, may also be at work in the bone spinal canal. Given that the single layer of dura that covers the spine is relatively avascular, contrast enhancement may actually reflect epidural venous enhancement. This conclusion is corroborated by the finding of enhancement in the characteristic location of epidural venous structures (that is, in the anterolateral spinal canal and sparing the midline and posterior). ${ }^{1}$

Data collected by Yousry, et al., ${ }^{51}$ further support these conclusions. A reciprocal relationship was found between the width of the dilated venous plexus and that of subdural fluid collections, consistent with the idea that venous engorgement and fluid collection are compensatory mechanisms to maintain intraspinal volume in the face of decreased CSF volume. Similarly, at spinal levels C-1 and $\mathrm{C}-2$, where venous compliance allows engorgement sufficient to maintain spinal volume, intraspinal fluid collections were never detected. At this location, transudated fluid is not necessary to maintain volume balance and therefore collects in the retrospinal region. Providing further evidence that fluid collections result from transudation and not leakage, these retrospinal fluid collections at $\mathrm{C}-1$ and C-2 were consistently detected in patients with ICH caused by LP. Cerebrospinal fluid leakage could explain these findings only if these patients already had spontaneous ICH with leaks all at the same level, C1-2, but without headache until after LP or if the LP had somehow caused a dural tear at $\mathrm{C} 1-2$. It is significant that resolution of spinal fluid collections on MR imaging parallels that of the postural headache. In one patient this retrospinal fluid collection persisted; his headache failed to resolve. It is also important to note that data collected by Raymackers, et al., ${ }^{43}$ has demonstrated a different time course for the development of cranial and spinal fluid collections.

In addition to its role in the diagnosis of $\mathrm{ICH}$, spinal MR imaging has been successfully used to reveal the location of CSF leaks. Unlike RC and CT myelography, MR imaging of the spine can yield positive results even if the CSF leakage is not active and can demonstrate CSF accumulation. ${ }^{28}$ By using heavily $\mathrm{T}_{2}$-weighted MR imaging, other components besides $\mathrm{H}_{2} \mathrm{O}$ (that is, CSF) could be de- leted from the image. ${ }^{28}$ Indeed, spinal MR imaging may succeed where RC and CT myelography fail to reveal the route of CSF leakage. ${ }^{27,40}$ In addition, MR imaging of the cervical cord has been used to demonstrate dilated perineural root sleeves and meningeal diverticula, abnormalities which have been shown to correspond to the site of such leaks. ${ }^{41}$ It is important to remember, however, that identifying such defects does not necessarily indicate that the site of leakage has been located. ${ }^{32}$

Spinal MR imaging has disadvantages, however. Nishizawa, et al., ${ }^{37}$ have asserted that results may depend on the body type of the patient and the level of CSF leakage, increasing false-positive or false-negative findings. Research has also brought into question the sensitivity of this technique for locating CSF leaks. According to data from one study, spinal MR imaging revealed the source of a CSF leak in only three of six patients. ${ }^{35}$

\section{Doppler Flow Imaging}

A new technique has recently been used to successfully diagnose $\mathrm{ICH} .{ }^{8}$ Given that the superior ophthalmic vein is a tributary of the cavernous sinus, Chen, et al., ${ }^{8}$ hypothesized that it might reflect the engorgement of the intracranial venous sinuses that occurs in ICH. Using transorbital color Doppler flow imaging, they demonstrated increased diameter and maximum flow velocity of the superior ophthalmic veins in patients with ICH. Statistical analysis indicates that this technique may have a very high sensitivity and specificity, demonstrating significant increases of these parameters in all patients ( 26 of 26 patients) with ICH when compared with either healthy volunteers or patients with headache of other origin. In addition, this change in blood flow reverted to normal after treatment, paralleling resolution of symptoms.

\section{PATHOPHYSIOLOGICAL FEATURES}

Two main theories have been proposed to explain the cause of headache in patients with ICH. Under normal conditions, the brain is supported by CSF, such that its weight of $1500 \mathrm{~g}$ amounts to only $48 \mathrm{~g}$ within the cranium. ${ }^{24}$ This remaining weight is supported by suspension from several pain-sensitive structures. These include the meninges, cerebral and cerebellar veins (tributaries of the sagittal and transverse sinuses, respectively) as well as the fifth, ninth, and 10th cranial nerves and the superior three cervical nerves. ${ }^{32}$ When CSF volume is depleted and its buoyant force is reduced, downward displacement of the brain causes traction on these supporting structures. Descent of the brain is exaggerated in the upright posture, which explains the orthostatic nature of the headache in ICH. Evidence for this theory is provided first by study data documenting downward displacement of cranial structures in patients with ICH (see earlier). Although descent has not been seen in all patients with the postural headache of ICH, such displacement may have been underestimated in incidence as well as severity because of supine positioning during MR imaging. Further evidence in support of this theory comes from data collected by Kunkle, et al., ${ }^{22}$ who induced postural headache in healthy patients by draining CSF. One of these individuals had previously undergone section of the roots of cranial nerves 
five and nine as well as the upper four cervical nerves on the left side, with analgesia in the regions to which these nerves project. After CSF drainage, this individual experienced headache only on the right side. The fact that headache associated with ICH rarely occurs in older patients, whose brain mass tends to be decreased, provides further support for this theory. ${ }^{29}$ Traction on spinal nerves could also explain the neck pain and radicular symptoms of the upper limb sometimes associated with ICH. Rupture of bridging veins is presumed to cause the subdural hematomas found in ICH (see earlier). It may also explain the red blood cells, pleocytosis, and high protein found in CSF obtained at LP in patients with ICH. ${ }^{39}$

This same mechanism also may cause cranial nerve palsies. Thus, traction on cranial nerves may explain hearing changes and dizziness (cranial nerve eight), horizontal diplopia (cranial nerve six), and facial numbness (cranial nerve five) or weakness (cranial nerve seven). Alternately, the auditory and vestibular symptoms may reflect changes in intralabyrinthine pressure. Lowering CSF pressure in cats causes a reduction in intralabyrinthine pressure and subsequent failure to transmit certain frequency tones, possibly by altering the pressure gradient across the cochlear aqueduct. ${ }^{9}$

Another theory posits dilation of intracranial vascular structures as the pathophysiological mechanism causing headache in patients with ICH. In the lateral recumbent position, healthy individuals have a mean CSF pressure of $150 \mathrm{~mm} \mathrm{H} \mathrm{H}_{2} \mathrm{O}$, approximately equal at all levels. With individuals in the erect posture, the pressure rises to 373 to $565 \mathrm{~mm} \mathrm{H}_{2} \mathrm{O}$ in the lumbar sac, becomes 0 at the level of the cisterna magna, and can fall to $-85 \mathrm{~mm} \mathrm{H}_{2} \mathrm{O}$ in the ventricles. ${ }^{50}$ Venous engorgement occurred in patients with $\mathrm{ICH}$, both intracranially as well as in the spine (see earlier). With application of the Monro-Kellie hypothesis, upright posture should be associated with further dilation of pain-sensitive intracranial venous structures, thus explaining the postural headache often experienced by patients with ICH. Maneuvers that decrease venous return to the heart (Valsalva, jugular venous compression, and coughing), and therefore increase intracranial venous volume, can reproduce headache in a patient with ICH even while positioned supine. ${ }^{39}$ This finding provides evidence that vascular dilation plays a role in the pathogenesis of headache in ICH. Such dilation might also cause diapedesis of cells and protein into the subarachnoid space, explaining the reticulocytes, xanthochromia, mononuclear pleocytosis, and increased protein found in CSF from patients with $\mathrm{ICH} .{ }^{39}$

This theory may also explain the pathogenesis of other symptoms of ICH. Pituitary hyperemia has been demonstrated on MR imaging studies in patients with ICH (see earlier). Resulting secretory dysfunction might explain the galactorrhea and hypopituitarism rarely associated with this syndrome. Similarly, engorgement of spinal epidural veins can cause nerve compression with resultant radiculopathy. ${ }^{1}$

It seems likely that both theories of pathogenesis contribute to the postural headache of ICH. Nonetheless, continued leakage of CSF after LP appears to be common whether a headache develops..$^{42}$ Furthermore, opening pressure on repeat LP may be low or even zero in patients without symptoms of $\mathrm{ICH}^{42}$ Similarly, study results have demonstrated a normal CSF opening pressure at LP in some patients with the syndrome of ICH. ${ }^{33}$ Taken together, these results could be explained by individual variation, not only in normal CSF pressures, but also in the pressure decrease required to produce symptoms. It is also possible that the absolute value of pressure is only part of the picture and that the rate of decrease is also important. Perhaps rapid changes in CSF volume overwhelm the homeostatic mechanisms that maintain CSF pressure.

\section{TREATMENT METHODS}

The headache of ICH typically resolves with conservative management and bed rest. It is believed that the supine position reduces CSF pressure at the site of leakage and therefore allows healing of the underlying meningeal defects. ${ }^{38}$ Intravenous or oral caffeine and theophylline have been reported to be dramatically effective in $75 \%$ of cases of ICH caused by LP. ${ }^{48}$ It has been proposed that methylxanthines produce arterial constriction through the blockade of adenosine receptors. ${ }^{26}$ Consequently, intracranial blood flow and, presumably, venous engorgement are decreased.

Other treatments aim at increasing CSF volume either by fluid restoration or by eliminating the leak. Strategies used in an effort to restore CSF volume include intravenous or oral hydration, increased salt intake, carbon dioxide inhalation, and steroid therapy. Few investigators have evaluated the efficacy of these empirical strategies, and therefore their role in the management of ICH remains unclear. Although some patients' clinical improvement has been coincident with glucocorticoid or mineralocorticoid treatment, other study results have shown such measures to be of questionable value. ${ }^{26,35}$ Furthermore, although these drugs cause systemic fluid retention, there is no convincing evidence indicating that steroid agents have any effect on CSF production or absorption..$^{41}$

When these measures fail, epidural blood patches are generally considered to be a safe and effective treatment. This technique was introduced by Gormley and is based on his observation that ICH follows LP less frequently when the tap is traumatic and bloody. ${ }^{42}$ It involves injection of autologous blood into the epidural space. Both immediate and lasting relief may be achieved, with the first patch being reported to be effective in 85 to $90 \%$ of cases. ${ }^{25}$ Repeat patches improve the efficacy to $98 \% .{ }^{6}$ The procedure is most effective if it is performed at or within one interspace of the leak. ${ }^{10}$ If the level of the leak is unknown, however, the blood can be injected into the lumbar epidural space and the patient's head lowered to $30^{\circ}$. The blood will slowly ascend to, and seal leaks in, the thoracic and cervical meninges. ${ }^{6}$ Research data indicate that this procedure may be effective over nine spinal segments. ${ }^{30}$ Complete resolution of headache is achieved less often in patients with multiple CSF leaks.9 Presumably, immediate relief results from a transient increase in CSF pressure, whereas lasting relief is provided by sealing of meningeal tears. ${ }^{24}$ Initially, the epidural blood forms a gelatinous tamponade over the dural hole that prevents 
leakage. Eventually, fibroblastic activity and collagen deposition result in scar formation, usually within 3 months. ${ }^{41}$ The most common complication of this procedure is back discomfort at the site of injection, occurring in one third of patients. ${ }^{42}$ Other complications include paresthesias, radiculopathy, and lumbosacral meningismus. ${ }^{6}$ Blood patches fail significantly more often when they are applied within 24 hours after LP. ${ }^{42}$

Alternatively, epidural infusion of saline may also yield immediate relief for patients with ICH. ${ }^{24}$ This procedure involves continuous infusion of saline for 2 to 3 days and, like the epidural blood patch, presumably works by causing an immediate increase in CSF pressure. Saline infusion need not occur at the site of leakage, however, thus avoiding the risks of cervical and thoracic epidural blood patches. ${ }^{41}$ Infusion of saline also avoids the risk of introducing blood into the subarachnoid space and resultant chemical meningitis. ${ }^{13}$ Saline infusion, however, is not as effective as an epidural blood patch and recurrences are significantly more common. ${ }^{42}$ These findings likely reflect the absence of clotting and scar formation that occur with the injection of blood. Other disadvantages of this procedure include a greater risk of infection due to the indwelling catheter and the hospitalization required for prolonged infusions. ${ }^{41}$ Overall, continuous epidural infusion of saline remains a useful strategy when other methods fail. ${ }^{13}$

Surgical correction may be required when all other measures have failed, especially if a dural tear or other meningeal defect has been demonstrated. In particular, meningeal diverticula can usually be treated by simple ligation, and complete resolution of headache has been achieved in up to $100 \%$ of patients. ${ }^{46}$ Surgical repair of meningeal tears has shown similar success..$^{35}$ In addition to the correction of meningeal defects, surgical drainage of subdural hematomas, a frequent complication of $\mathrm{ICH}$, also may be necessary. ${ }^{24}$

A new technique, epidural injection of fibrin glue, has shown encouraging preliminary results. ${ }^{36}$

\section{CONCLUSIONS}

The patient presenting with headache is often a diagnostic dilemma, and the indiscriminate screening of these patients by performing MR imaging is not a good use of an expensive resource. Correlation of the headache with the patient position, a recent history of LP, or an accompanying cranial nerve deficit should trigger a suspicion of $\mathrm{ICH}$ and prompt the appropriate use of Gd-enhanced MR imaging of the brain. The headache of ICH usually resolves with conservative management, but if it does not, epidural blood patching is highly effective.

\section{References}

1. Albayram S, Wasserman BA, Yousem DM, et al: Intracranial hypotension as a cause of radiculopathy from cervical epidural venous engorgement: case report. AJNR 23:618-621, 2002

2. Ali SA, Cesani F, Zuckermann JA, et al: Spinal-cerebrospinal fluid leak demonstrated by radiopharmaceutical cisternography. Clin Nucl Med 23:152-155, 1998

3. Alvarez-Linera J, Escribano J, Benito-Leon J, et al: Pituitary enlargement in patients with intracranial hypotension syndrome. Neurology 55:1895-1897, 2000
4. Bell WE, Joynt RJ, Sahs AL: Low spinal fluid pressure syndromes. Neurology 10:512-521, 1960

5. Benamor M, Tainturier C, Graveleau P, et al: Radionuclide cisternography in spontaneous intracranial hypotension. Clin Nucl Med 23:150-151, 1998

6. Brightbill TC, Goodwin RS, Ford RG: Magnetic resonance imaging of intracranial hypotension syndrome with pathophysiological correlation. Headache 40:292-299, 2000

7. Bruera O, Bonamico L, Giglio JA, et al: Intracranial hypotension: the nonspecific nature of MRI findings. Headache 40: 848-852, 2000

8. Chen CC, Luo CL, Wang SJ, et al: Colour Doppler imaging for diagnosis of intracranial hypotension. Lancet 354:826-829, 1999

9. Chung SJ, Kim JS, Lee MC: Syndrome of cerebral spinal fluid hypovolemia: clinical and imaging features and outcomes. Neurology 55:1321-1327, 2000

10. Davenport RJ, Chataway SJ, Warlow CP: Spontaneous intracranial hypotension from a CSF leak in a patient with Marfan's syndrome. J Neurol Neurosurg Psychiatry 59:516-519, 1995

11. Evan RW, Mokri B: Spontaneous intracranial hypotension resulting in coma. Headache 42:159-160, 2002

12. Fishman RA, Dillon WP: Dural enhancement and cerebral displacement secondary to intracranial hypotension. Neurology 43:609-611, 1993

13. Gibson BE, Wedel DJ, Faust RJ, et al: Continuous epidural saline infusion for the treatment of low CSF pressure headache. Anesthesiology 68:789-791, 1988

14. Good DC, Ghobrial M: Pathologic changes associated with intracranial hypotension and meningeal enhancement on MRI. Neurology 43:2698-2700, 1993

15. Grant R, Condon B, Hart I, et al: Changes in intracranial CSF volume after lumbar puncture and their relationship to post-LP headache. J Neurol Neurosurg Psychiatry 54:440-442, 1991

16. Han S, Kim Y, Kim Y, et al: A case report of unexpected clinical course of spontaneous intracranial hypotension. J Kor Neurol Assoc 13:129-133, 1995 (Reference unverified)

17. Hochman MS, Naidich TP: Diffuse meningeal enhancement in patients with overdraining, long-standing ventricular shunts. Neurology 52:406-409, 1999

18. Hong M, Shah GV, Adams KM, et al: Spontaneous intracranial hypotension causing reversible frontotemporal dementia. Neurology 58:1285-1287, 2002

19. Inenaga $C$, Tanaka $T$, Sakai N, et al: Diagnostic and surgical strategies for intractable spontaneous intracranial hypotension. Case report. J Neurosurg 94:642-645, 2001

20. Koss SA, Ulmer JL, Hacein-Bey L: Angiographic features of spontaneous intracranial hypotension. AJNR 24:704-706, 2003

21. Kraemer G, Hopf H, Eissner D: CSF hyperabsorption: a cause of spontaneous low CSF pressure headache. Neurology 37 (Suppl 1):238, 1987 (Abstract)

22. Kunkle E, Ray B, Wolff H: Experimental studies on headache: analysis of the headache associated with changes in intracranial pressure. Arch Neurol Psychiatry 49:323-358, 1943 (Reference unverified)

23. Labadie EL, van Antwerp J, Bamford CR: Abnormal lumbar isotope cisternography in an unusual case of spontaneous hypoliquorrheic headache. Neurology 26:135-139, 1976

24. Lay C, Campbell K, Mokri B: Low cerebrospinal fluid headache, in Goadsby PJ, Silberstein SD (eds): Headache. Boston: Butterworth-Heinemann, 1997, pp 355-367 (Reference unverified)

25. Liebold RA, Yealy DM, Coppolla M, et al: Post-dural-puncture headache: characteristics, management, and prevention. Ann Emerg Med 22:1863-1870, 1993

26. Marcelis J, Silberstein SD: Spontaneous low cerebrospinal fluid pressure headache. Headache 30:192-196, 1990 
27. Matsumara A, Anno I, Kimura H, et al: Diagnosis of spontaneous intracranial hypotension by using magnetic resonance myelography. Case report. J Neurosurg 92:873-876, 2000

28. Matsumara A, Anno I, Nose T, et al: Intracranial hypotension. J Neurosurg 95:914-916, 2001

29. Messori A, Simonetti B, Regnicolo L, et al: Spontaneous intracranial hypotension: the value of brain measurements in diagnosis by MRI. Neuroradiology 43:453-461, 2001

30. Moayeri NN, Henson JW, Schaefer PW, et al: Spinal dural enhancement on magnetic resonance imaging associated with spontaneous intracranial hypotension. Report of three cases and review of the literature. J Neurosurg 88:912-918, 1998

31. Mokri B: Spontaneous cerebrospinal fluid leaks: from intracranial hypotension to cerebrospinal fluid hypovolemia-evolution of a concept. Mayo Clin Proc 74:1113-1123, 1999

32. Mokri B, Atkinson JL, Dodick DW, et al: Absent pachymeningeal gadolinium enhancement on cranial MRI despite symptomatic CSF leak. Neurology 53:402-404, 1999

33. Mokri B, Hunter SF, Atkinson JL, et al: Orthostatic headaches caused by CSF leak but with normal CSF pressures. Neurology 51:786-790, 1998

34. Mokri B, Parisi JE, Scheithauer BW, et al: Meningeal biopsy in intracranial hypotension: meningeal enhancement on MRI. Neurology 45:1801-1807, 1995

35. Mokri B, Piepgras DG, Miller GM: Syndrome of orthostatic headaches and diffuse pachymeningeal gadolinium enhancement. Mayo Clin Proc 72:400-413, 1997

36. Mokri B, Posner JB: Spontaneous intracranial hypotension: the broadening clinical and imaging spectrum of CSF leaks. Neurology 55:1771-1772, 2000

37. Nishizawa S, Tanaka T, Inenaga C: Intracranial hypotension. J Neurosurg 95:915, 2001 (Letter)

38. Nosik WA: Intracranial hypotension secondary to lumbar nerve sleeve tear. JAMA 157:1110-1111, 1955

39. Pannullo SC, Reich JB, Krol G, et al: MRI changes in intracranial hypotension. Neurology 43:919-926, 1993

40. Rabin BM, Roychowdhury S, Meyer JR, et al: Spontaneous intracranial hypotension: spinal MR findings. AJNR 19: 1034-1039, 1998

41. Rando TA, Fishman RA: Spontaneous intracranial hypotension: report of two cases and review of the literature. Neurology 42: 481-487, 1992
42. Raskin NH: Lumbar puncture headache: a review. Headache 30:197-200, 1990

43. Raymackers JM, Duprez T, Jeanjean A, et al: Discrepant time course of cranial and spinal subdural collections in a case of SIH treated by EBP. Eur Radiol 11:2310-2313, 2001

44. Renowden SA, Gregory R, Hyman N, et al: Spontaneous intracranial hypotension. J Neurol Neurosurg Psychiatry 59: $511-515,1995$

45. Roll JD, Larson TC III, Soriano MM: Cerebral angiographic findings of spontaneous intracranial hypotension. AJNR 24: 707-708, 2003

46. Schievink WI, Meyer FB, Atkinson JL, et al: Spontaneous spinal cerebrospinal fluid leaks and intracranial hypotension. J Neurosurg 84:598-605, 1996

47. Schievink WI, Reimer R, Folger WN: Surgical treatment of spontaneous intracranial hypotension associated with a spinal arachnoid diverticulum. Case report. J Neurosurg 80:736-739, 1994

48. Sechzer P, Abel L: Post-spinal anesthesia headache treated with caffeine. Curr Ther Res Clin Exp 24:307-312, 1978 (Reference unverified)

49. Sharma P, Sharma A, Chacko AG: Syringomyelia in spontaneous intracranial hypotension. Case report. J Neurosurg 95: 905-908, 2001

50. Silberstein SD, Marcelis J: Headache associated with abnormalities in intracranial structure or pressure, in Dallessio DJ, Silberstein SD (eds): Wolff's Headache and Other Head Pain, ed 6. New York: Oxford University Press, 1993, pp 438-461 (Reference unverified)

51. Yousry I, Forderreuther S, Moriggl B, et al: Cervical MR imaging in postural headache: MR signs and pathophysiological implications. AJNR 22:1239-1250, 2001

Manuscript received September 28, 2003.

Accepted in final form November 4, 2003.

Address reprint requests to: Michael S. Tenner, M.D., Department of Radiology, New York Medical College, Valhalla, New York 10595. email: TennerM@WCMC.com. 